\title{
Conjunctival flap surgery in the management of ocular surface disease (Review)
}

\author{
MIHAIL ZEMBA $^{1 *}$, ALINA-CRISTINA STAMATE ${ }^{1 *}$, CALIN PETRU TATARU $^{1}$, \\ DANIEL CONSTANTIN BRANISTEANU ${ }^{2}$ and FLORIAN BALTA ${ }^{1}$
}

\author{
${ }^{1}$ Department of Ophthalmology, 'Carol Davila' University of Medicine and Pharmacy, Bucharest 050474; \\ ${ }^{2}$ Department of Ophthalmology, 'Grigore T. Popa' University of Medicine and Pharmacy, Iasi 700115, Romania
}

Received May 15, 2020; Accepted June 16, 2020

DOI: $10.3892 /$ etm.2020.8964

\begin{abstract}
Literature regarding conjunctival flap surgery was reviewed to describe and discuss the rationale for this type of procedure. The conjunctival flap is an acknowledged surgery for the treatment of various corneal diseases with a chronically compromised ocular surface, such as severe dry eye, neurotrophic or neuroparalytic disease, or bullous keratopathy. The purpose of this surgery is to restore the integrity of the corneal surface and thus to prevent gradual corneal ulceration and secondary infection, as well as to ameliorate pain, reduce the need for frequent medications, improve cosmetic appearance, and offer an alternative to invasive surgery or enucleation. Since the introduction of more effective methods of treating severe ocular surface diseases, conjunctival flap surgery has rarely been the primary modality of treatment and has usually followed a range of medical and surgical treatments. The availability of improved ocular lubricants, more effective antimicrobials, bandage contact lenses, tissue adhesives, and other corneal and conjunctival surgical interventions, has reduced the need for conjunctival flaps. However, conjunctival flaps remain extremely useful in selected cases and deserve a place in the ophthalmologist's repertoire for the management of ocular surface disease.
\end{abstract}

\section{Contents}

1. Introduction

2. Purpose and therapeutic effects

3. Indications

4. Surgical techniques

Correspondence to: Professor Daniel Constantin Branisteanu, Department of Ophthalmology, 'Grigore T. Popa' University of Medicine and Pharmacy, 16 University Street, Iasi 700115, Romania E-mail: dbranisteanu@yahoo.com

*Contributed equally

Key words: conjunctival flap surgery, pedicle conjunctival flap, severe ocular surface disease, refractory corneal ulcer, corneal perforation

\section{Complications}

6. Conclusions

\section{Introduction}

Conjunctival flap (CF) surgery has an acknowledged role in assuring a stable ocular surface and repressing inflammation in compromised corneas or eyes with reduced visual potential (1).

In 1958, Gundersen reintroduced and popularized the procedure of using a thin total $\mathrm{CF}$ to repair the cornea in a variety of ocular surface diseases (2). Before the refinement of surgical supplies and techniques, almost all perforating corneal injuries were sealed by drawing a hood type flap over the laceration, after a careful excision of the prolapsed uveal tissue. His technique extended the usage of CF from mainly the treatment of injuries, especially perforating corneal injuries, to other ocular surface diseases.

CF surgery is still a benchmark surgery and although its use has highly decreased in developed countries after the emergence of therapeutic penetrating keratoplasty, amniotic membrane transplantation and epithelial transplantation techniques, it should be taken into consideration in selected cases. These alternative treatments constitute considerable technological progress, but have some impediments, especially concerning their limited availability in developing countries, more specifically the deficit of corneal donor tissue for corneal transplantation (3).

\section{Purpose and therapeutic effects}

The main purpose of this surgery is to recover the integrity of the corneal surface to prevent gradual corneal ulceration and secondary infection, as well as to achieve several other secondary effects: relieving pain, reducing topical medications, enhancing the aesthetic appearance and providing an option to invasive surgery or enucleation (3-10).

The therapeutic effects of CF surgery are probably promoted by several mechanisms:

i) The conjunctival flap covers the affected corneal tissue and prevents tears, proteolytic enzymes, and proinflammatory mediators from reaching the corneal ulcer and causing stromal lysis $(11,12)$. 
ii) Supplies the compromised corneal tissue with vascularized tissue that defends the cornea from more damage; the rich supply of blood and lymphatic vessels provides nutrients, including cellular components and growth factors, that increase the resistance to infection and anticollagenolytic substances that suppress stromal ulceration (12-14).

iii) On the other hand, peritomy decreases the vascular supply and so the amount of circulating antibodies to the area of ulceration.

iv) Relieves pain in bullous keratopathy because bullae no longer appear on the surface of the cornea.

v) Reduces corneal sensitivity so that a scleral shell prosthesis can be used (11).

\section{Indications}

The original indications defined for this procedure included herpetic keratitis, bullous keratopathy, neuroparalytic keratopathy, and traumatic relapsing keratopathy $(1,2)$, but many of these diseases are treated at present with other medical or surgical therapies.

In herpetic necrotizing stromal keratitis unresponsive to topical and systemic antivirals, a CF may be the only efficient method to control pathologic inflammation, possibly by replacing the diseased stromal bed with healthy basal tissue on which a stable epithelium can evolve (15) and by supplying blood vessels and anti-inflammatory cellular components and cytokines to the stromal bed to help in suppressing infective, inflammatory, and ulcerative processes $(16,17)$. For such severe cases, Gao et al (18) evaluated the efficiency of $\mathrm{CF}$ surgery combined with medical therapy, after removal of the necrotic tissue, and all patients recorded ocular surface stabilization. Fluoresceine staining revealed that ocular surface epithelialization was obtained after approximately 3 days. They concluded that $\mathrm{CF}$ is useful for patients with deep corneal ulcers, but recommended the amniotic membrane in superficial ones (18).

In bullous keratopathy, the $\mathrm{CF}$ forms a complete and uniform surface over the exposed corneal nerve endings and alleviates corneal pain, and at the same time acts as a partial osmotic barrier at the tear film-epithelium interface (3). Patients with glaucomatous bullous keratopathy devote an enormous period of time and effort to their eye disease. Contrary to what is expected, a significant proportion of medications is directed towards corneal pain and not to pain from high intraocular pressure. After surgery, due to the formation of a stable ocular surface, many of the patients require less or no eye medication and also fewer office visits. An important aspect to emphasize is the significant improvement in the patient's lifestyle after flap surgery (16).

In noninfectious corneal afflictions, such as neuroparalytic keratopathy, recruiting of neutrophils and the release of collagenases are essential stages in disease pathogenesis. It has been demonstrated that vascularized tissues are very resistant to ulceration and perforation because of anticollagenolytic substances released from the circulation that arrest these destructive effects (15). Gundersen flaps were primarily conducted in cases with reduced visual potential. The main goals in these situations were to obtain alleviation and to avoid enucleation, which can cause considerable psychological trauma. By placing a CF, patients can preserve their eyes and maintain an adequate cosmetic appearance, a goal that can be improved by using a $\mathrm{CF}$ as thin as possible. In some patients, a flap provides a surface for the fitting of a cosmetic contact lens or scleral shell. Some advocate that a proper examination of opaque blind eyes is difficult and that prophylactic enucleation should be performed because they may harbor malignancy (16).

The list of indications has expanded over time to include chronic infectious keratitis and tectonic indications such as peripheral marginal ulcerations and corneal melts $(7,16,19)$. Corneal ulcers refractory to medical treatment can rapidly progress to a corneal perforation that can lead to debilitating visual consequences, so rehabilitation of the integrity of the ocular surface is mandatory to conserve the visual function (6).

CFs are usually not successful in cases of active refractory bacterial and fungal corneal ulcers. However, in emergency situations, especially in countries that lack cornea donors, CF surgery can be employed as a temporary measure for a few months, to inhibit disease progression and to maintain globe integrity, until a secondary surgical intervention can be performed for vision restoration. Some studies have shown that $\mathrm{CF}$ can be a useful alternative in these situations and can offer a better prognosis for a potential future corneal transplantation. Nizeyimana et al (20) obtained a good postoperative outcome for all their patients with refractory fungal keratitis, with or without perforation, with control of the infection and preservation of the globe. Moreover, the study performed by Zhong et al (21), that combined full-thickness conjunctival flap surgery with amniotic membrane transplantation in the treatment of severe fungal keratitis without perforation, showed $88.24 \%$ of eyeball preservation. Abdulhalim et al (14) compared the results of bipedicle $\mathrm{CF}$ and cryopreserved amniotic membrane graft in the treatment of non-viral infectious keratitis resistant to medical treatment. Successful results were observed in 18 out of 20 eyes (90\%) in each group. Their results indicated that $\mathrm{CF}$ and amniotic membrane transplantation are efficient as a surgical treatment for infectious keratitis because they could recover ocular surface integrity and offer metabolic and mechanical support for corneal healing. The use of amniotic membrane can have a benefit as it does not hinder corneal examination like $\mathrm{CF}$, it does not affect limbal stem cells, and supplementarily, it conserves the conjunctiva which may be required for another ocular surgery.

Conjunctival surgery was also attempted in the treatment of peripheral autoimmune-related ulcerative corneal diseases, such as Mooren's ulcer and peripheral ulcerative keratitis due to granulomatosis with polyangiitis (previously known as Wegener's granulomatosis) and rheumatoid arthritis (22).

Although CF has proved to be a simple, effective, short-term treatment for managing corneal perforation or impending perforation in infectious corneal ulcers, it is not recommended for Mooren's ulcer. A case report by Chen et al (23) of a relapsing Mooren's ulcer after multi-layered amniotic membrane transplantation combined with conjunctival autografting from the opposite healthy eye highlighted this conclusion. In their case, conjunctival grafting appeared to cause rapid relapsing of Mooren's ulcer, probably by encouraging blood vessel growth and displaying stromal antigen to the systemic circulation. Similar results were obtained in another study, which 
described two cases of Mooren's ulcer in which CF surgery was performed instead of conjunctival autografting. CF appeared to produce a fast aggravation of the ulcer and a subsequent spread to the entire cornea. Histopathological examination of Mooren's ulcers proved that the conjunctival tissues next to the corneal ulcer harbored a great number of immune effect cells and circulating antibodies. The initiation of local autoimmune processes additionally stimulated the conjunctiva to produce enzymes, including collagenase and proteolytic enzymes. Consequently, the conjunctiva acts as a reservoir for immune mediators and enzymes during the pathogenesis of Mooren's ulcers, which can elucidate why Mooren's ulcers always commence in the peripheral cornea and also why they can be successfully treated using conjunctival resection, freezing, and thermocoagulation (24). However, Lu et al (25) reported pain relief and stable ocular condition in a patient with peripheral ulcerative keratitis and necrotizing scleritis in granulomatosis with polyangiitis treated surgically with a combination of removal of necrotic tissue, cryotherapy and $\mathrm{CF}$ surgery. Another small case series by Awan and Ramaesh (26) also showed good recovery with the restoration of vision after superior forniceal advancement conjunctival pedicle in two patients with corneal perforation and impending perforation due to rheumatoid arthritis.

It is still unclear whether CF could be recommended to treat corneal perforation due to immune-mediated corneal ulcerative diseases, such as granulomatosis with polyangiitis and rheumatoid arthritis-associated corneal ulcers.

\section{Surgical techniques}

The original technique described by Gundersen suffered a variety of modifications over time, in an effort to overcome its disadvantages and enhance the success rate of CFs. Other methods have also been used to preserve globe integrity and include buccal mucous membrane grafts, dermal grafts, and Tenon's capsule grafts (15).

During the past decades, advancement in microsurgical technique has enabled the use of a selective pediculate CF sutured in place and adjusted to cover the area of the affected cornea. This microsurgical approach offers an appropriate option to the method developed by Gunderson without its deficiencies. The steps to an efficient pedicle CF include choosing the proper site and size, movement of the flap without traction, conserving the flap's blood supply, avoiding cautery and conjunctival or corneal epithelial entrapment, debridement and application of povidone-iodine to infected ulcers, securing the flap over the denuded area with running 10-0 monofilament nylon sutures, and avoiding excessive traction on sutures (27). The pedicle CF can also supply further support in the healing of a corneal ulcer. Depending on the depth of the ulcer, it can be thin (without Tenon's capsule) in superficial ulcers or thick (with Tenon's capsule) in deep ulcers (12).

Several studies have emphasized the advantages of partial CF surgery and outlined that the selection of a specific type of $\mathrm{CF}$ is decided by the size and location of the corneal lesion.

The types of CFs described across literature are the following:

i) Total conjunctival flap described by Gundersen is the most commonly used CF and is a thin, bipedicle, bridge flap that involves a 360-degree peritomy, debridement of the entire corneal epithelium and mobilization of the conjunctiva at the superior fornix to cover the entire corneal surface (11). Disadvantages: Difficulties in monitoring the corneal disease and measuring intraocular pressure to detect glaucoma development, fornix shortening, ptosis, corneal opacification, conjunctivalization of the cornea, extensive corneal vascularization (9), disturbance of the local architecture, possibly leading to limbal stem cell deficiency (13) and affecting the conjunctiva that may be necessary for glaucoma filtering surgery $(8,14)$.

ii) Bipedicle bridge flap (bucket-handle) is used for small central or paracentral corneal lesions that do not need coverage of the entire cornea and involves a 180-degree peritomy, separation of the conjunctiva from the underlying Tenon's capsule, an incision parallel to the limbus and mobilization of the conjunctiva over the corneal ulcer (12). Advantages: assessing the remaining uncovered cornea and anterior chamber.

iii) Single pedicle flap (racquet flap) is a smaller racquet-shaped perilimbal $\mathrm{CF}$ that is mobilized to cover perilimbal corneal lesions. Advantages: less prone to retraction.

iv) Advancement flap is used for paralimbal lesions, usually in conjunction with a lamellar corneal patch or scleral patch graft, and involves performing a peritomy and pulling the adjacent conjunctiva over the peripheral corneal lesion (4). Disadvantages: the tendency to retract over time.

Sandinha et al (6) described a different CF technique referred to as superior forniceal conjunctival advancement pedicle (SFCAP) for managing corneal perforations or impending corneal perforations. Corneal perforations are usually associated with inflammation of the perilimbal conjunctiva, which is friable and difficult to dissect, making SFCAP a more reliable option, because it uses peripheral non-inflamed conjunctiva. The technique implies the creation of a pedicle, which includes a prominent blood vessel, by making two parallel conjunctival incisions, placing its advancing edge on the cornea and suturing it with 10-0 nylon interrupted sutures around the corneal ulcer. The advantages of this technique are a lesser extent of conjunctival dissection, that does not affect the local conjunctiva in case of later glaucoma surgery and rapid healing that eliminates the stimulus for vasogenic substances and prevents the formation of neovessels in the peripheral corneal which is advantageous for future corneal grafts.

Another approach was described by Sharma et al (13) and presented a new concept, in which the $\mathrm{CF}$ was repositioned at its original site, in a patient that did not show any improvement in symptoms after the initial surgery for refractory fungal corneal ulcer. Early repositioning of the CF after healing of the cornea is beneficial as the prolonged presence of the $\mathrm{CF}$ on the cornea increases the risk of ocular surface damage. CF promotes corneal vascularization and the surgery itself can induce limbal stem cell loss that may lead to an unstable ocular surface, which may necessitate limbal autograft surgery. Both conditions present an increased risk of graft rejection if penetrating or lamellar keratoplasty is considered at a later stage (9). Considering the intraoperative technique, it should be taken into account that the dissected flap is very thin and that it may undergo necrosis with sloughing of the conjunctiva in cases of necrotic ulcers. This may be possible 
because of the poor vascularity of the perilimbal conjunctival tissues and in such cases rigorous corneal debridement should be performed (6). Removal of the necrotic tissue is supposed to reduce the antibody-producing inflammatory cells adjacent to the ulcer and enhance the absorption of anti-inflammatory and immune-modulatory agents (25).

A study by Sun et al (28) proved that it is safe and effective to remove necrotic corneal tissue before CF surgery under the guidance of AS-OCT in the treatment of fungal keratitis. The AS-OCT could more accurately measure the range and depth of ulcers under the corneal edema than the slit lamp microscope. Thus, they could avoid the incomplete removal of the affected corneal tissue and prevent postoperative recurrence of fungal infection and they could also better distinguish between the ulceration and the infiltration area to avoid removing too much healthy corneal tissue and to extend the healing time of the corneal ulcer.

Regarding tissue removal, deep lamellar keratectomy combined with CF surgery has also been tried to aid management of the infection and to alleviate pain, with great results, as proved by Cremona et al (29) in a case series of two patients diagnosed with Acanthamoeba keratitis. The goal of deep lamellar keratectomy combined with $\mathrm{CF}$ is to remove the infected tissue in order to decrease the number of microorganisms and to eliminate necrotic tissue. Also, the CF brings conjunctival vessels and lymphatic channels that increase the immune response against the amoeba.

Adding cryotherapy to flap surgery may have a cumulative effect. Cryotherapy of the corneal surface at temperatures of -50 to $-60^{\circ} \mathrm{C}$ not only denatures the pathogen's cell walls but can also remove part of the antigen-antibody complexes and decrease inflammation caused by the area of ulceration and neovascularization $(8,25)$.

In an attempt to increase the efficiency of CF surgery, fibrin glue has been used as an adhesive to maintain the flap in place. A study by Chung and Mehta (30) confirmed that fibrin glue application is a feasible option to sutures in CF surgery. It minimizes surgical time, offers faster ocular surface recovery, less postoperative pain, and a good cosmetic result, with minimal scarring after resorption.

\section{Complications}

CF can be plagued by both intraoperative and postoperative complications. Most of these are avoidable by careful case selection, preoperative evaluation of the area to be maneuvered and meticulous care to instrumentation and surgical technique (4).

A considerable area of healthy conjunctiva is necessary and that limits its use in ocular surface diseases with significant conjunctival involvement or in eyes with previous retinal or glaucoma surgery.

Suturing is one of the most essential steps of this procedure. The flap over the cornea should not be too tight or too loose. Sutures that are too tight may cut through a tight $\mathrm{CF}$ and a loose $\mathrm{CF}$ may not heal and detach in the postoperative period (29,31).

Complications after CF surgery are relatively uncommon. The most frequently reported complications include flap retraction, conjunctival buttonholes and erosions, epithelial inclusion cysts, corneal perforations (3), and occasionally ptosis (2). However, positioning of a CF over the cornea has some disadvantages: Hindering the visualization of intraocular structures, compromising the penetration of topical medications (e.g., glaucoma medications) and a poor cosmetic result in case of thick flaps. Also, as mentioned before, removal of limbal epithelium harboring stem cells can possibly affect the stability of the ocular surface and this should be considered in cases in which flap removal and subsequent keratoplasty are planned.

Another potential and serious problem is the persistence of infection under the flap; also, perforation under the flap in herpetic stromal keratitis has been reported (3). These complications and disadvantages should be balanced against the multiple benefits of CF surgery.

\section{Conclusions}

CF surgery is a simple, efficient, and cost-effective method of treatment for ocular surface disease resistant to medical therapy. The goal of this surgery is to re-establish the integrity of the corneal surface, as well as to relieve pain, reduce the need for frequent medications, enhance aesthetic appearance, and provide an alternative to invasive surgery or enucleation. In some instances, it can also be used as a method of temporizing future corneal transplantation.

CFs are underused because of the availability of alternative therapeutic options including bandage contact lenses, amniotic membrane transplantation, epithelial transplantation and therapeutic keratoplasty, that have replaced them in many cases. These options do not lack complications and limitations. Donor corneal tissue is frequently not available. Amniotic membrane handling requires specialized equipment and involves a slim risk of infectious disease transmission. Extended wear bandage contact lenses are expensive, usually do not remove the need for medication, and are associated with an increased risk of microbial keratitis.

Taking into consideration its low complication rate and its proven immediate and sustained outcome, we believe that CF surgery should be considered by ophthalmologists in the management of the resistant ocular surface disease.

\section{Acknowledgements}

Not applicable.

\section{Funding}

No funding was received.

\section{Availability of data and materials}

Not applicable.

\section{Authors' contributions}

MZ and ACS had a substantial contribution to the conception and design of the study, as well as the drafting of the manuscript. ACS and DCB performed the literature research and contributed to the acquisition and analysis of the data. 
DCB, CPT and FB did a critical interpretation of the data for the study and edited the manuscript. All authors revised the manuscript for important intellectual content, read and approved the final version of the manuscript to be published. All authors have agreed to be accountable for all aspects of the work in ensuring that questions related to the accuracy or integrity of any part of the work are appropriately investigated and resolved.

\section{Ethics approval and consent to participate}

Not applicable.

\section{Patient consent for publication}

Not applicable.

\section{Competing interests}

The authors declare that they have no competing interests.

\section{References}

1. Gundersen T and Pearlson HR: Conjunctival flaps for corneal disease: Their usefulness and complications. Trans Am Ophthalmol Soc 67: 78-95, 1969.

2. Gundersen T: Conjunctival flaps in the treatment of corneal disease with reference to a new technique of application. AMA Arch Opthalmol 60: 880-888, 1958.

3. Lim LS, How AC, Ang LP and Tan DT: Gundersen flaps in the management of ocular surface disease in an Asian population. Cornea 28: 747-751, 2009.

4. Mannis MJ: Conjunctival flaps. Int Ophthalmol Clin 28: 165-168, 1988.

5. Adesina OO, Vickery JA, Ferguson CL and Stone DU: Stromal melting associated with a cosmetic contact lens over a Boston keratoprosthesis: Treatment with a conjunctival flap. Eye Contact Lens 39: e4-e6, 2013.

6. Sandinha T, Zaher SS, Roberts F, Devlin HC, Dhillon B and Ramaesh K: Superior forniceal conjunctival advancement pedicles (SFCAP) in the management of acute and impending corneal perforations. Eye (Lond) 20: 84-89, 2006.

7. Mauger TF and Craig E: Combined acanthamoeba and Stenotrophomonas maltophilia keratitis treated with a conjunctival flap followed by penetrating keratoplasty. Cornea 25: 631-633, 2006.

8. Shah A, Talati M and Mauger T: Medical and surgical management of Pasteurella canis infectious keratitis. IDCases 9: 42-44, 2017.

9. Deshmukh R, Stevenson LJ and Vajpayee R: Management of corneal perforations: An update. Indian J Ophthalmol 68: 7-14, 2020.

10. Jhanji V, Young AL, Mehta JS, Sharma N, Agarwal T and Vajpayee RB: Management of corneal perforation. Surv Ophthalmol 56: 522-538, 2011.

11. van Rij G, Rinkel-van Driel B, Beekhuis WH and Renardel de Lavalette JG: Conjunctival transplantation. Doc Ophthalmol 67: 145-150, 1987.

12. Stamate AC, Tătaru CP and Zemba M: Update on surgical management of corneal ulceration and perforation. Rom $\mathbf{J}$ Ophthalmol 63: 166-173, 2019.

13. Sharma A, Mohan K, Sharma R and Nirankari VS: Repositioning of pedicle conjunctival flap performed for refractory corneal ulcer. Middle East Afr J Ophthalmol 21: 89-91, 2014.
14. Abdulhalim BE, Wagih MM, Gad AA, Boghdadi G and Nagy RR: Amniotic membrane graft to conjunctival flap in treatment of non-viral resistant infectious keratitis: A randomised clinical study. Br J Ophthalmol 99: 59-63, 2015.

15. Tuli SS, Schultz GS and Downer DM: Science and strategy for preventing and managing corneal ulceration. Ocul Surf 5: 23-39, 2007.

16. Alino AM, Perry HD, Kanellopoulos AJ, Donnenfeld ED and Rahn EK: Conjunctival flaps. Ophthalmology 105: 1120-1123, 1998.

17. Filip-Ciubotaru F, Manciuc C, Stoleriu G and Foia L: NADPH Oxidase: Structure and activation mecanisms (Review). Note I. Rev Med Chir Soc Med Nat Iasi 120: 29-33, 2016.

18. Gao H, Jia Y, Li S, Wang T, Tan Y and Shi W: Conjunctival flap covering combined with antiviral and steroid therapy for severe herpes simplex virus necrotizing stromal keratitis. ScientificWorldJournal 2015: 565964, 2015.

19. Zhou Q, Long X and Zhu X: Improved conjunctival transplantation for corneal ulcer. Zhong Nan Da Xue Xue Bao Yi Xue Ban 35: 814-818, 2010 (In Chinese).

20. Nizeyimana H, Zhou DD, Liu XF, Pan XT, Liu C, Lu CW and Hao JL: Clinical efficacy of conjunctival flap surgery in the treatment of refractory fungal keratitis. Exp Ther Med 14: 1109-1113, 2017.

21. Zhong J, Wang B, Li S, Deng Y, Huang H, Chen L and Yuan J: Full-thickness conjunctival flap covering surgery combined with amniotic membrane transplantation for severe fungal keratitis. Exp Ther Med 15: 2711-2718, 2018.

22. Brănişteanu DE, Pintilie A, Andreş LE, Dimitriu A, Oanţă A, Stoleriu G and Brănişteanu DC: Ethiopatogenic hypotheses in lichen planus. Rev Med Chir Soc Med Nat Iasi 120: 760-767, 2016.

23. Chen KH, Hsu WM and Liang CK: Relapsing Mooren's ulcer after amniotic membrane transplantation combined with conjunctival autografting. Ophthalmology 111: 792-795, 2004.

24. Li S, Deng Y, Du C, Huang H, Zhong J, Chen L, Wang B and Yuan J: Rapid deterioration of Mooren's ulcers after conjunctival flap: A review of 2 cases. BMC Ophthalmol 17: 93, 2017.

25. Lu CW, Zhou DD, Wang J and Hao JL: Surgical treatment of peripheral ulcerative keratitis and necrotizing scleritis in granulomatosis with polyangiitis. Saudi Med J 37: 205-207, 2016.

26. Awan MA and Ramaesh K: Superior forniceal advancement conjunctival pedicle (SFACP) in the management of corneal perforations and impending perforations due to rheumatoid arthritis related autoimmune corneal melts. J Pak Med Assoc 60: 129-131, 2010.

27. Khodadoust A and Quinter AP: Microsurgical approach to the conjunctival flap. Arch Ophthalmol 121: 1189-1193, 2003.

28. Sun GH, Li SX, Gao H, Zhang WB, Zhang MA and Shi WY: Clinical observation of removal of the necrotic corneal tissue combined with conjunctival flap covering surgery under the guidance of the AS-OCT in treatment of fungal keratitis. Int J Ophthalmol 5: 88-91,2012.

29. Cremona G, Carrasco MA, Tytiun A and Cosentino MJ: Treatment of advanced acanthamoeba keratitis with deep lamellar keratectomy and conjunctival flap. Cornea 21: 705-708, 2002.

30. Chung HW and Mehta JS: Fibrin glue for Gundersen flap surgery. Clin Ophthalmol 7: 479-484, 2013

31. Grange JD, Duquesne N, Roubeyrol F, Branisteanu D, Sandon K, Fleury J, Gerard JP, Chauvel P, Pinzaru G, Jean-Louis B, et al: Double irradiation for macroscopic radioresistance or recurrence of melanomas of the posterior uvea: Clinical, ballistic, therapeutic and prognostic aspects. Series of 19 cases among 462 patients. J Fr Ophtalmol 22: 1054-1063, 1999 (In French).

This work is licensed under a Creative Commons Attribution-NonCommercial-NoDerivatives 4.0 International (CC BY-NC-ND 4.0) License. 